\title{
Recurrent Neural Network Based MPPT Control of Grid Connected DFIG for Wind Turbine
}

\author{
MOHSEN DAVOUDI, AMIN KASIRI FAR \\ Department of Electrical Engineering \\ Imam Khomeini International University \\ University Blvd., Qazvin, \\ IRAN \\ davoudi@eng.ikiu.ac.ir
}

\begin{abstract}
This paper presents a new maximum-power-point-tracking (MPPT) controller in wind power generation using artificial neural networks (ANN) in order for making the wind turbine function in optimum working point and get high efficiency of wind energy conversion at different conditions. The algorithm uses fully connected recurrent neural network and is trained online using real-time recurrent learning (RTRL) algorithm in order to avoid the oscillation problem in wind-turbine generation systems. It generates control command for speed of the rotor side converter using optimal algorithm to enable the control system in order to track the maximum power point. The rotor speed and wind-turbine torque are the inputs of the networks, and the command signal for the rotor speed of wind turbine is the output. Simulation results verify the performance of the proposed algorithm.
\end{abstract}

Key-Words: - wind power generation, induction generator, recurrent neural network, vector control, real-time learning

Received: June 27, 2019. Revised: December 14, 2019. Accepted: January 7, 2020.

Published: January 17, 2020.

\section{Introduction}

Since the fossil fuels gradually exhausting and concerns about environmental pollution continuously rising, the usage of the renewable energies has become important in the power networks. Renewable energy sources like wind, photovoltaic, and bio-fuel cells can be interfaced to the power network using step-up converter systems for a high power transmission [1-3].

Due to the promising developments in the wind power generators as well as the advances in power electronic components and their applications in highpower systems, wind energy becomes inevitable and more interesting in electrical power applications. The current studies in this area for increasing the windturbine generator efficiency and MPPT methods up to now can be classified into three categories:

1)Tip-speed ratio (TSR) method discussed in [4-6], is used to tune the generator rotational velocity in order to reach to an optimum TSR value in which the velocity of rotor and wind velocity are either measured or computationally estimated [28].

2)Power signal feedback (PSF) method studied in [7-9], requires the prior information about the curve of the wind turbine maximum power in order to be given to an intelligent control system for tracking.
3)Hill-climb searching (HCS) method discussed in [10-18], is an on-line algorithm in which the peak power point of the wind turbine is being continuously searched and delivered at the output side. The HCS algorithm includes the observation and perturbation control methods too. It has been often used for the MPPT problem because of its simplicity for implementation [16-18].

In this paper, a new method has been proposed that using MPPT control algorithm deals with the variable wind speed. This method has been developed based on Fully connected recurrent neural networks that includes only one hidden layer. The neuron weights of the network layers are being continuously adjusted by real-time recurrent learning (RTRL) algorithm, which is more suitable for on-line continuous training compared to the off-line learning algorithms like back propagation learning algorithm. The tracking controller developed in this paper is an algorithm based on a HCS type control, in which the control system searches for the maximum power point during its functioning $[28,29]$.

The inputs of the network are the rotor velocity and wind turbine torque, hence, there is no requirement for anemometer and wind velocity measurement for control system. A doubly fed induction generator (DFIG) wind turbine has been chosen to implement the new MPPT algorithm. Simulation results show 
the performance of the proposed method which is trained online using real-time recurrent learning (RTRL) algorithm.

The next sections of the paper is organized as follows: the modelling of the wind turbine has been performed in the second section; The third section discusses the field-oriented control applied on DFIG system; forth section presents the control strategy for DFIG-based wind turbine with MPPT; The structure of the proposed recurrent neural network has been presented in section five and the simulation results and discussion have been organized in section six.

\section{Wind Turbine Modelling}

The actual mechanical power output of a wind turbine can be written as [19]

$$
P_{m}=\frac{1}{2} \rho \pi R{ }^{2} V_{W}{ }^{3} C_{P}(\lambda \beta)
$$

where, $\mathrm{R}$ is the wind turbine blade radius, $\mathrm{V}_{\mathrm{W}}$ is the wind velocity, $\rho$ is the air density and $C_{P}$ is the coefficient of performance. $C_{P}$ is a function of tip speed ratio, $\lambda$, and blade pitch angle, $\beta$. The tip speed ratio is defined as

$$
\text { (22) }=\frac{\omega_{r} R}{V_{W}}
$$

where, $\omega_{r}$ is the the turbine rotor angular velocity of in $\mathrm{rad} / \mathrm{s}$. $C_{P}$ can be calculated using the relation

$$
C_{P}=05176\left[\frac{116}{\lambda_{i}}-04 \beta-5\right] e^{\frac{-21}{\lambda_{i}}}+00068 \lambda
$$

where

$$
\lambda_{i}=\frac{1}{\frac{1}{\lambda+008 \beta}+\frac{6035}{1+\beta^{3}}}
$$

Fig. 1 shows the power coefficient $C_{P}$ as the function of tip speed ratio $\lambda$. The power coefficient is at its maximum value at a certain value of tip speed ratio that is called optimum tip speed ratio $\lambda_{\text {opt }}$. It is the goal to reach to the maximum possible power generation for which the turbine should always operate at $\lambda_{\text {opt }}$. Control of the turbine rotational velocity to the optimum speed of rotation leads to reach the maximum power generation [28]. Fig. 1 implies the $P_{M}-\omega_{r}$ curves at different wind velocities as shown in Fig. 2.

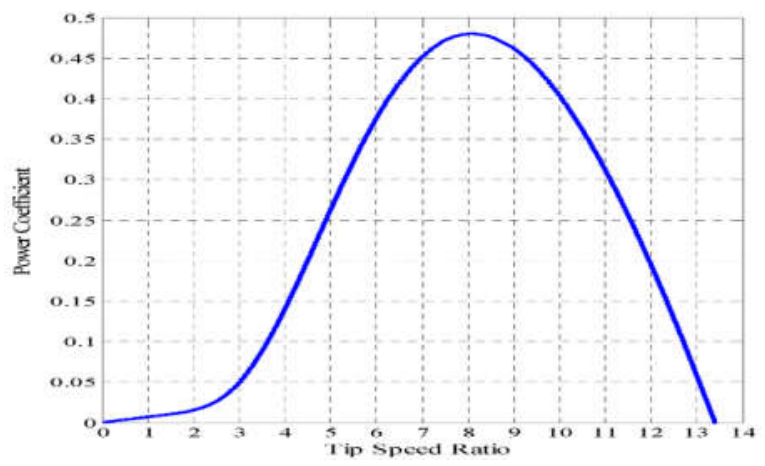

Fig. 1: Power coefficient curve in terms of tip speed ratio

As shown in Fig. 2 at the maximum point in various wind velocity, we have

$$
\underset{(5)}{\partial \omega_{R}}=0
$$

In this study concerning (5) a new adaptive MPPT algorithm has been proposed which employs an artificial neural network that contains the information of wind-turbine torque, and rotational velocity as the inputs of the recurrent neural networks has been developed. The wind-turbine torque and the rotational velocity are involved in the output power equation as $(6)[26,32]$.

$$
P_{m} 6 \neq T_{m} \omega_{r}
$$

Thus, getting the maximum value of $T_{m} \omega_{r}$ will lead to generating maximum power at each specific wind velocity.

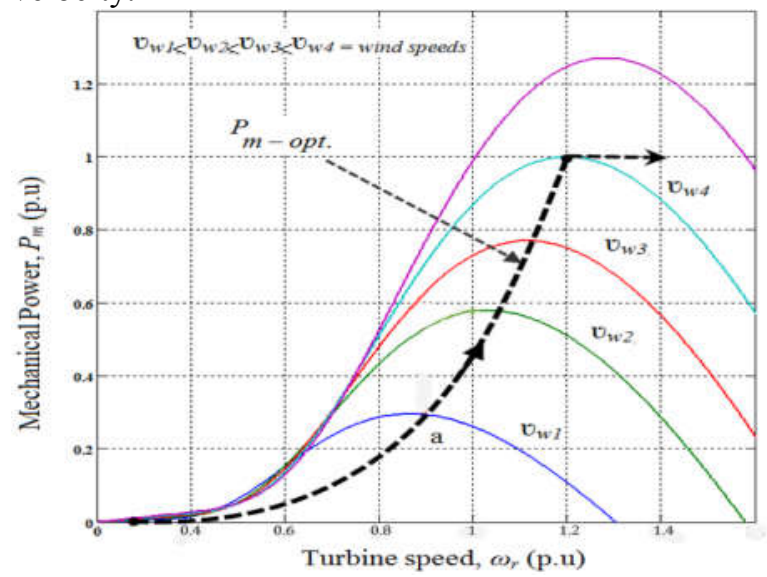

Fig. 2: Mechanical Power vs. turbine speed 


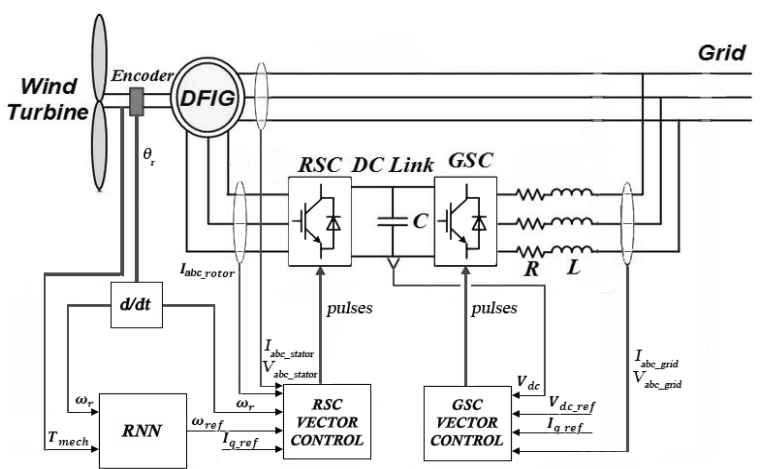

Fig. 3: DFIG Wind Turbine and control scheme

\section{Field-oriented control for DFIG System}

A DFIG-based wind turbine generally consists of an induction machine with wounded rotor, wind turbine, rotor side converter (RSC), grid side converter (GSC) and DC-link capacitor. The block diagram of the turbine and control system has been shown in Fig. 3. The wound rotor induction machine stator winding is basically connected to the power network directly while the rotor winding is connected to the network via $\mathrm{AC} / \mathrm{DC} / \mathrm{AC}$ power converter in which the IGBTs are functioning. The power converter consists of a couple of components: the grid-side converter (GSC) and the rotor-side converter (RSC) with a DC capacitor placed between [30].

\subsection{Control system of rotor side converter}

The rotor side converter in stator-voltage oriented frame has been vector controlled. The stator-voltage oriented frame is utilized for the controller design, in which the d-axis and the q-axis rotor current components are for active and reactive power (or voltage) control separately. After comparison of the rotor d-current and q-current reference values to the real rotor $\mathrm{d}$ and $\mathrm{q}$ currents, error signals pass through PI controllers to maintain the $\mathrm{d}-\mathrm{q}$ voltage control signals in the power converter [20].

The ANN-based MPPT controller, proposed in this paper, generates $\omega_{\text {ref }}$, the reference speed which when compared to rotor rotational speed, produces the rotor d-axis current reference value via a PI controller. Hence, maximum power will be extracted from wind turbine.

\subsection{Grid side converter control system}

The grid-side power converter controls the DC-link voltage by stabilizing it constant in order to generate or (possibly) absorb reactive power. The DC-link voltage is the d-q reference frame oriented along the grid stator voltages, enabling independent active and reactive power control that flow between the grid and the converters. The actual DC-link voltage is compared with reference value $V_{d c-r e f}$.

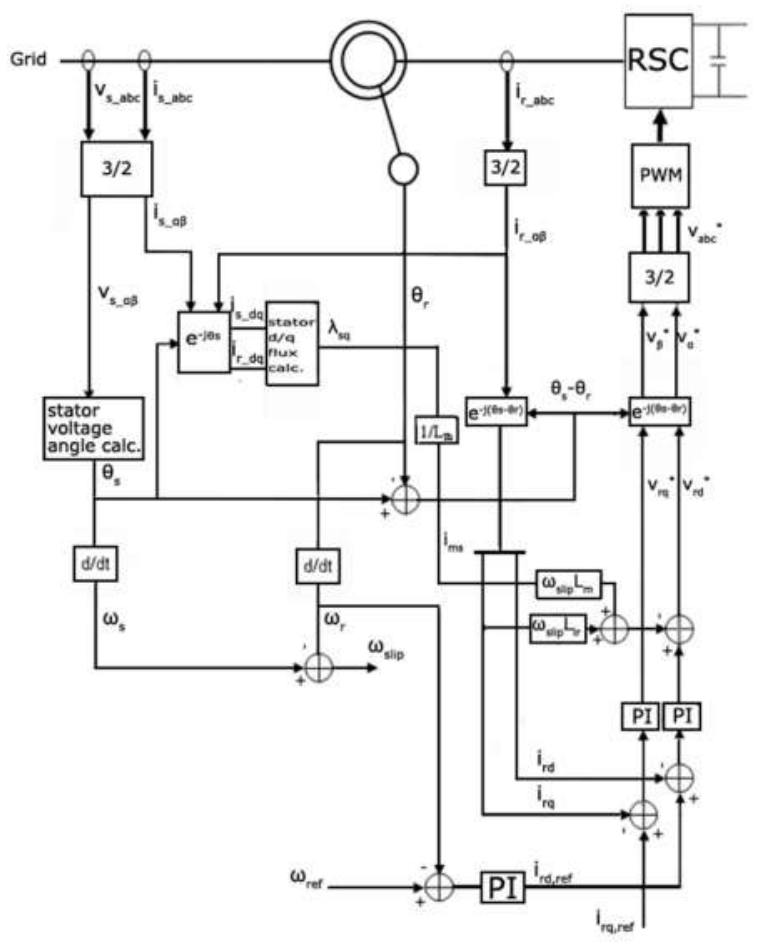

Fig. 4: Rotor side vector control

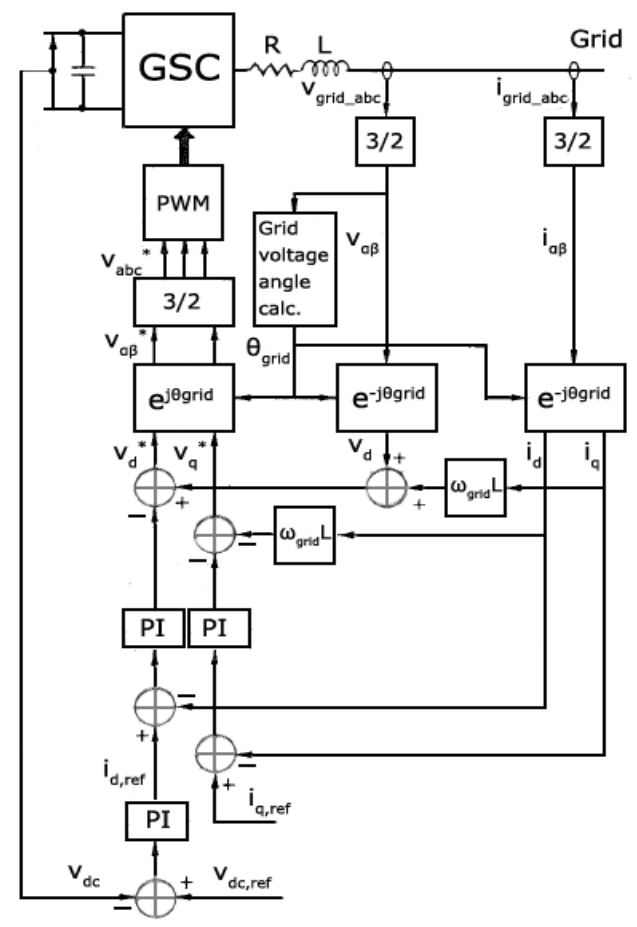

Fig. 5: Grid side vector control

The difference between these couple of values will be given to a couple of PI controllers designed for 
generation of the reference value of d-axis grid voltage. On the other hand, the difference between the actual reactive power and the reference value $Q_{r e f}$ is going to be given to another couple of PI controllers in order to generate the reference value of the q-axis grid voltage $[21,30]$.

Typical block diagrams of RSC and GSC vector control systems are demonstrated in Fig. 4 and Fig. 5 , respectively. For brevity and to avoid proposing unnecessary details, vector controllers formulation hasn't discussed in this study $[22,23]$.

\section{The MPPT control strategy for DFIG-based wind turbines}

In a DFIG-based power wind turbine whereas only a part of total power passes through rotor connection to the grid, the power converter handles a fraction of the power between $20 \%-30 \%$. That is why the losses of the power electronic in the power converter is very low comparing a system in which the converter handles all the power. It has the advantage of needing smaller and cheaper back-to-back converters compared to singly-fed machines, but the rotor speed can only vary by a limited amount, typically $30 \%$ either side of synchronous speed. In this study, bounds to this operational rotor speed range are called $\omega_{\mathrm{r}-\text { mifnd }} \omega \mathrm{r}$-max essential values.

Typically $v_{w-c u t i n}$ and $v_{w-n o m i n a l}$ wind velocities are chosen to correspond with above-mentioned essential rotor speeds by adjusting required gearbox ratio [22]. Fig. 6 shows how the mechanical power varies with the wind velocity for a wind turbine with variable-speed, characterized by 4 wind velocities, cut-in, nominal, rated and cut-out. Below cut-in wind velocity curve, there is not enough torque exerted by the wind force on the rotating turbine blades, or in another case of very slow rotation, power generated is less than power losses. The increment of the speed cause the wind turbine begin to generate electrical power.

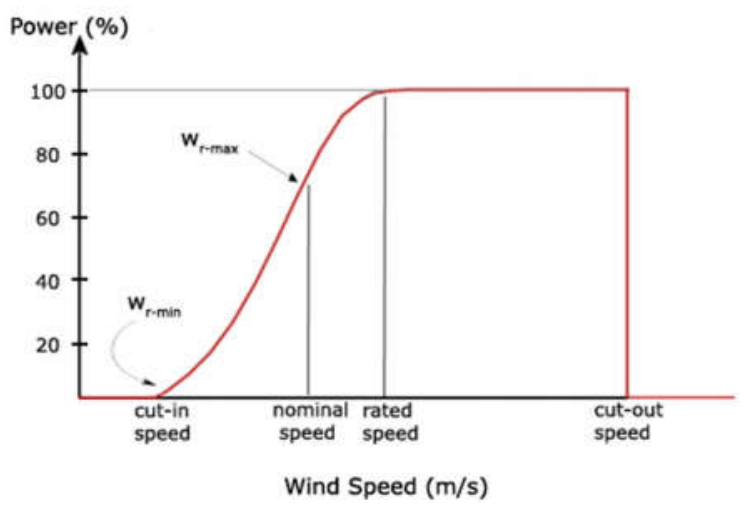

Fig. 6: Mechanical power in terms of wind velocity.
In order to keep generator at its controllable range, converters have to keep rotor speed at $\omega_{\mathrm{r}-\max }$ for wind velocities between nominal and rated values. Rated wind velocity is where the generator produces its rated power. Above the rated wind velocity pitch adjustment should be applied in order to limit the attainable wind power to its maximum.

Optimizing (6) via genetic algorithm leads to the cube of wind velocities and rotor speeds, which maximize $C_{p}(\lambda \beta)$. As shown in Fig. 7, the relationship between these values, is linear. Thus, according to (1), (3) and (6)

$$
\begin{aligned}
& P_{\text {m-max }}=\frac{1}{2} \rho \pi R{ }^{2} V_{W}{ }^{3} C_{P-\max }\left(\lambda_{\text {opt }} 0\right) \\
& (T \omega)_{\max }=\frac{1}{2} \rho \pi R^{2} V_{W^{3}} C_{P-\max }\left(\lambda_{\text {opt }} 0\right. \text { (ß) }
\end{aligned}
$$

any specific rotor speed in controllable range can be mapped onto its corresponding $(T \omega)_{\max }$.

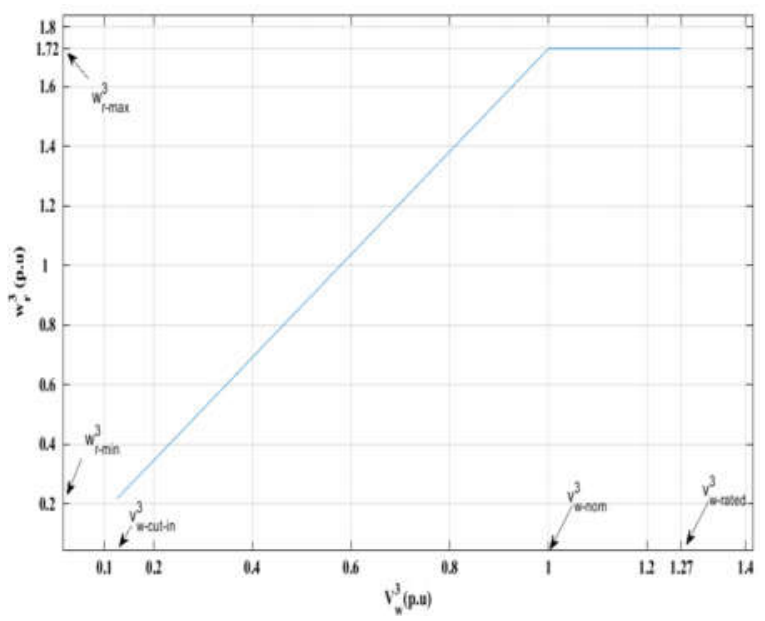

Fig. 7: Optimal rotor speed cubed which lead to $C_{p-\max }$ as a function of wind velocity cubed.

\section{The structure of the proposed recurrent neural network}

Recurrent (interactive) neural networks are the networks that can have signal flow in both directions by employing feedbacks in the neural network links. Recurrent networks are powerful enough to dial with complicated problems. The outputs of the earlier computations are fed back into the neural network again, that makes them to have a kind of memory effect. The neural networks with feedback have dynamic states that change continuously to reach a stable state. The network states remain stable until new inputs makes them change and reach a new stable point [31].

Online learning, which is applied to proposed recurrent network, is used when data becomes 
available gradually in a sequential order to map the data set to the corresponding meaning. It has the advantage that does not need to wait until all of the training data to be collected. The on=line training uses the arrived signal as the new training sample to be used to train the network. It causes the network getting trained gradually during it function.

In this case, online learning eliminates the necessity of measuring wind velocity by anemometers, which are imprecise and have some other disadvantages like the distortion of wind velocity in relation to precipitation.

Fig. 8 shows the layout of a fully connected recurrent neural network which is implemented in this paper. $\omega_{r}$ and $T_{\text {mech }}$ represent external input signals, which are rotational speed of rotor and wind-turbine mechanical torque respectively, and $\omega_{\text {ref }}$ represents output signal which is rotor speed reference command.

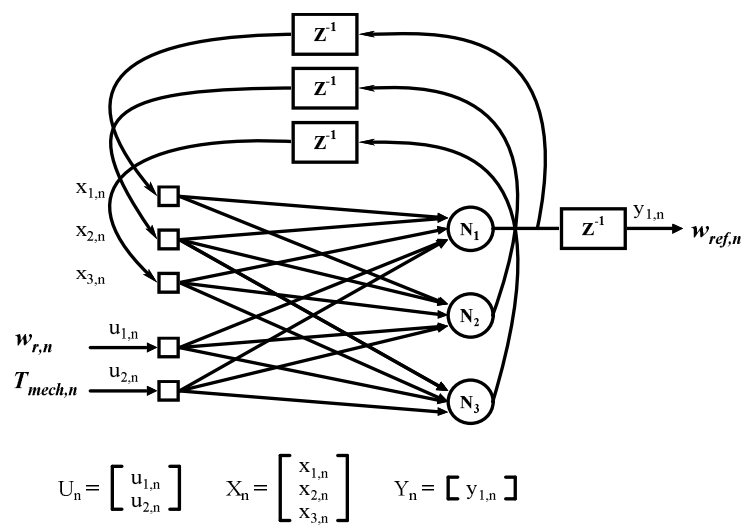

Fig. 8: Fully connected recurrent network [24].

As seen in Fig. 8, the state of a nonlinear discretetime system has been denoted by 3-by-1 vector $\mathbf{X}_{\mathrm{n}}$. in the same way, the input applied to the system has been denoted by the 2-by-1 vector $\mathbf{U}_{\mathrm{n}}$ and the corresponding output of the system has been denoted by the 1-by-1 vector $\mathbf{Y}_{\mathrm{n}}$ [24], [31]. the system (state) equation of the model for $n=0,1,2, \ldots$ is

$$
X_{n+1}=\Phi_{\mathrm{n}}\left(W_{a n} X_{n}+W_{b n}\left(\mathscr{U}_{n}\right)\right.
$$

And the measurement equation of the model is

$$
Y_{k}(1 \theta) W_{C} X_{n}
$$

where $\mathbf{W}_{\mathrm{a}}$ is a 3-by-3 matrix representing the weights of the 3 neurons of the hidden layer that are feeding back to the input layer, $\mathbf{W}_{\mathrm{b}}$ is a 3-by-2 matrix representing the weights of the hidden neurons that are connected directly to the source nodes of the input layer [24].
To simplify the composition of (9), from the state model the use of bias has been excluded. $\mathbf{W}_{\mathrm{c}}$ is a 1by-3 matrix representing 1 linear neuron weights in output layer that is connected directly to the hidden layers. The output layer does not have the bias for simplification reasons[24]. $\Phi \cdot R^{q} \rightarrow R^{q}$ is a diagonal map described by

$$
\Phi:\left[\begin{array}{l}
x_{1} \\
x_{2} \\
x_{3}
\end{array}\right] \rightarrow\left[\begin{array}{c}
\varphi\left(x_{1}\right) \\
1 \varphi\left(x_{2}\right) \\
\varphi\left(x_{3}\right)
\end{array}\right]
$$

The function $\varphi(x)$ which is a nonlinear function, represents the sigmoidal logistic activation function of the hidden neurons.

$$
\varphi(x)=(12)^{1}
$$

So, the proposed neural network has a couple of neuron layers: a concatenated input-feedback layer and a processing layer of computation nodes. Also, the connections of the neurons include both feed forward links and feedback links [24]. (9) can be written in another form as

$$
X_{n+1}=\left[\begin{array}{c}
\varphi\left(w_{1}^{T} \xi_{n}\right) \\
\varphi\left(w_{2}^{T} \xi_{n}\right) \\
\varphi\left(w_{3}^{T} \xi_{n}\right)
\end{array}\right] X_{0}=\left(\begin{array}{r}
0 \\
00) \\
0
\end{array}\right]
$$

where the 5 -by- 1 vector $w_{j}$ is the neuron weight vector $j$ which is

$$
\left.w_{j}=\left[\begin{array}{l}
w_{a j} \\
w_{b j}
\end{array}\right] 12 B 4\right)
$$

where $w_{a j}$ and $w_{b j}$ are the $j^{\text {th }}$ column in the weight matrices that is transposed $\mathbf{W}_{a}^{T}$ and $\mathbf{W}_{b}^{T}$, respectively [24]. And the 5-by-1 vector $\xi_{n}$ is defined by

$$
\xi_{n}\left( \pm 5\left[\begin{array}{l}
x_{1 n} \\
x_{2 n} \\
x_{3 n} \\
\omega_{r n} \\
T_{m n}
\end{array}\right]\right.
$$

\subsection{RTRL algorithm and weight updating rule}

The RTRL algorithm has the capability that the weights of the fully connected recurrent network is being adjusted, in real-time while the network is in function and continues to perform signal processing [24]. The adjustment that has been applied to the weight vector $w_{j n}$ of neuron $j$ for $n=0,1,2, \ldots$ is determined by

$$
\Delta w_{j n}=\eta T_{m n} W_{c} \Lambda_{j n} \dot{\rho}_{\bar{n}}=12(16)
$$


where

$$
W_{c}=[1,0,0]
$$

where $\eta$ is learning rate, $e_{n}$ is 1 -by-1 error vector, $T_{m n}$ is wind-turbine torque and $\Lambda_{j n+1}$ is calculated independently by

$$
\begin{gathered}
\Lambda_{j n+1}=\Phi_{n}\left(W_{a n} \Lambda_{j n}+Z_{j n}\right) \Lambda_{j 0}=0 \\
(1 \phi)=123
\end{gathered}
$$

where, as mentioned before

$$
W_{a n}=\left[\begin{array}{ll}
w_{11} W_{12} W_{13} \\
w_{21} W_{22} w_{23} \\
w_{31} w_{32} w_{33}
\end{array}\right]
$$

$w_{i j}$ is the weight connecting $x_{\dot{m}}$ to the neuron $j$. The definitions of $\Lambda_{j n} Z$. ${ }_{j n}$ and $\Phi_{n}$ are given in following descriptions, respectively

1. $\Lambda_{j n}$ is a 3-by-5 matrix defined as the partial derivative of the state vector $\mathbf{X}_{\mathrm{n}}$ with respect to the weight vector $w_{j}$ :

$$
\Lambda_{j n}\left(z 00 \frac{\partial X_{n}}{\partial w_{j}}\right.
$$

2. $Z_{j m}$ is a 3-by-5 matrix in which the rows are zero except for the $j^{\text {th }}$ row, that is equal to the vector $\xi_{n}^{T}$ transpose

$$
Z_{j n}=\left[\begin{array}{c}
0 \\
\xi_{n}^{T} \\
0
\end{array}\right] \rightarrow \text { jthrowj }=123
$$

3. $\Phi_{n}$ is a 3-by-3 matrix which is diagonal and its $j^{\text {th }}$ diagonal element equals to the partial derivative of its activation function; thus

$\Phi_{n}=\operatorname{diag}\left(\varphi^{\prime}\left(w_{1}^{T} \xi_{n}\right) \varphi^{\prime}\left(w_{2}^{T} \xi_{n}\right) \varphi \varphi^{\prime}\left(w_{3}^{T} \xi_{n}\right)(22)\right.$

\subsection{Tracking Error}

In this study, the 1-by-1 error vector is defined as

$$
\begin{aligned}
e_{n} & =T_{m n} \omega_{\text {refn }}-(T \omega)_{\operatorname{maxn}} \\
& \left.=T_{m n} W_{c} X_{n}-(T \omega)_{\max (23)} 3\right)
\end{aligned}
$$

The sum of squared errors (SSE) at time-step $n$ is defined in as a function of $e_{n}$ by

$$
\varepsilon_{n}\left(\frac{1}{4}\right) e_{n}^{T} e_{n}
$$

Instantaneous estimate of the gradient has been used for training the recurrent network in real-time, that leads to an approximation to the steepest descent method. (24) has been considered as the cost function that has to be minimized, and differentiated with respect to the weight vector $w_{j}$, and, using the chain rule of calculus, (25) is obtained [24, 25].

$$
\frac{\partial \varepsilon_{n}}{\partial w_{j}}=T_{m n} W_{c} \Lambda_{j n} \dot{\boldsymbol{\rho}}_{\bar{n}}=12(25)
$$

which leads to weight update rule formulation, (16), afore-mentioned.

\subsection{Estimating Maximum T $\omega$}

$(T \omega)_{\operatorname{maxn}}$ is achieved by a simple single-layer feed forward ANN with a single input, one output and a single hidden layer with 15 neurons which trained offline using input and target datasets showing in Fig. 9. $\boldsymbol{m}$ and $\boldsymbol{n}$ are arbitrary number of elements. Based on mentioned idea in section IV, that linearity between optimal rotor speed and wind velocity both cubed, in controllable range $\left( \pm 30 \% \Delta \omega_{s}\right)$, can lead to map $\omega_{r}$ onto $(T \omega)_{\max }$.

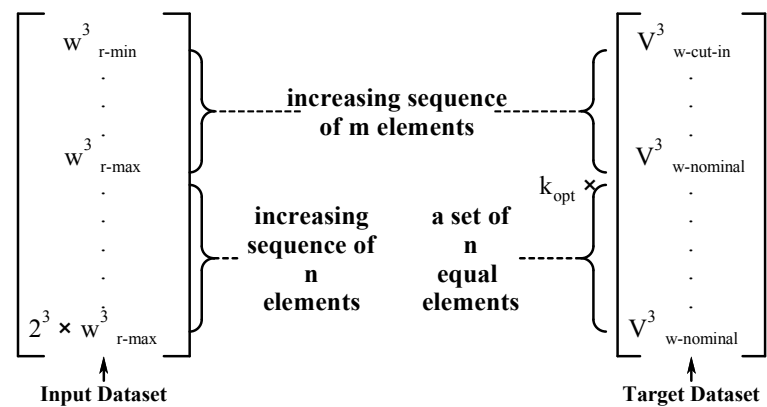

Fig. 9: Input and target datasets for training feed-forward ANN.

$k_{\text {opt }}$ is defined as

$$
k_{\text {opt }}=\frac{1}{2} \rho A C \text { (26) ax }
$$

According to (7) and (8), $k_{o p t} v_{w}^{3}$ is equal to maximum possible value of $T_{m} \omega_{r}$ for a particular value of wind velocity. As inferred from (23) the difference between the $T_{m} \omega_{\text {ref }}$ and $(T \omega)_{\max }$ is used to update the ANN weights to generate $\omega_{\text {ref }}$.

The ANN MPPT is based on an idea that for all values of the wind velocities the error between the $T_{m} \omega_{\text {ref }}$ and $(T \omega)_{\max }$ reaches to the minimum when the generator operates at its maximum working power point. When the wind turbine operates at other operating points (not necessarily at the maximum 

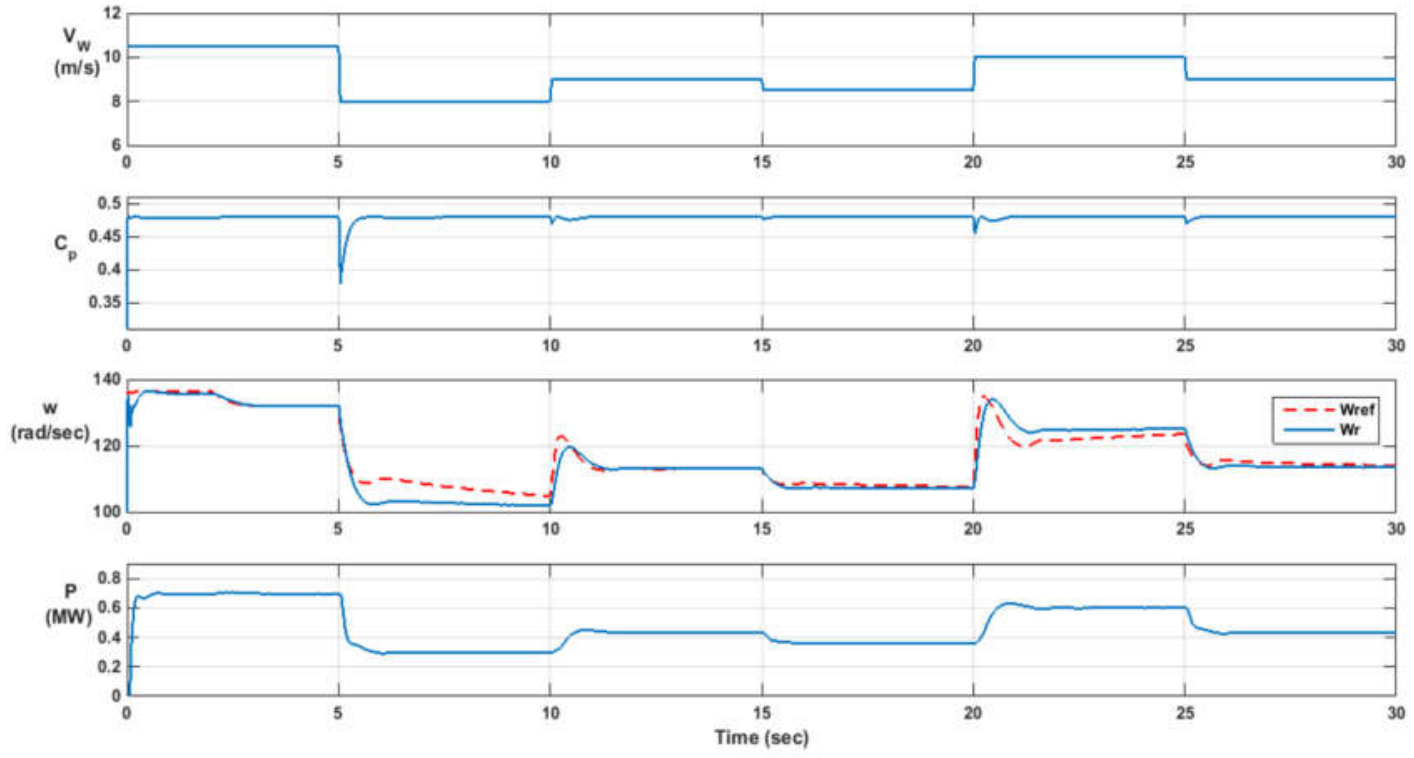

Fig. 11: Simulation results in the case when sudden changes applied.

point), the weights of recurrent neural networks will be modified accordingly. The new weights of RNN helps the MPPT controller to generate control command for reference speed to reduce the error and moves the system to maximum power point. The block diagram of the proposed MPPT controller has been demonstrated in Fig. 10.

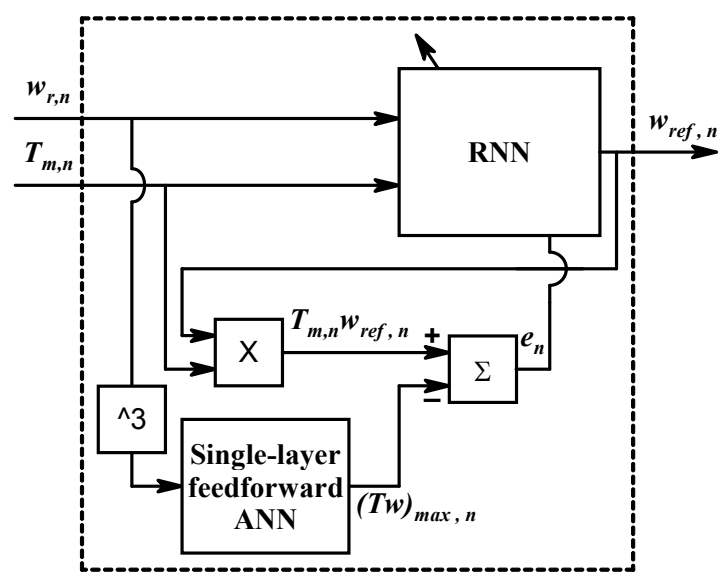

Fig. 10: ANN MPPT controller structure

\section{Simulation Results}

To illustrate the effectiveness of the proposed controller, simulation is carried out using MATLAB/Simulink simulation tool. The important parameters of the power generation system has been presented in Table 1. A rectangular, a sinusoidal and a noisy wind velocity profile has been applied to the wind turbine as shown in Fig. 11, Fig. 12 and Fig. 13, respectively. These figures show the wind velocity, power coefficient, rotor speed, reference rotor speed (dash line), and generated active power during the operation of the wind turbine.

Table 1: Important parameters of the power generation system

\begin{tabular}{|l|l|}
\hline Parameter & Value \\
\hline Rated power & $1.5 \mathrm{MW}$ \\
\hline Rated stator voltage & $575 / 995 \mathrm{~V}(\mathrm{D} / \mathrm{Y})$ \\
\hline Synchronous speed & $1200 \mathrm{rpm}$ \\
\hline Nominal wind velocity & $12 \mathrm{~m} / \mathrm{s}$ \\
\hline Maximum $\mathrm{C}_{\mathrm{p}}$ & 0.48 \\
\hline Optimal $\lambda$ & 8.1 \\
\hline
\end{tabular}

Results of simulation show suitable performance of the proposed MPPT controller. As seen, the performance coefficient of the wind turbine, $\mathrm{C}_{\mathrm{p}}$, is almost at its maximum value of 0.48 for the wind velocity while sudden changes have been applied. As shown in Fig. 12, with sinusoidal wind velocity profile with variable amplitude, the proposed MPPT controller makes the performance coefficient $\mathrm{Cp}$ to be kept nearly at 0.48 that is the maximum value. Fig. 13 shows a noisy wind velocity profile which is similar to real wind profiles, proposed controller shows suitable performance keeping the performance coefficient $\mathrm{Cp}$ being kept fairly at its maximum value.

As can be seen in Fig. 14, the tracking error is getting close to minimum value (zero) despite of the variations in wind velocity applying rectangular, sinusoidal and noisy profiles, respectively. 

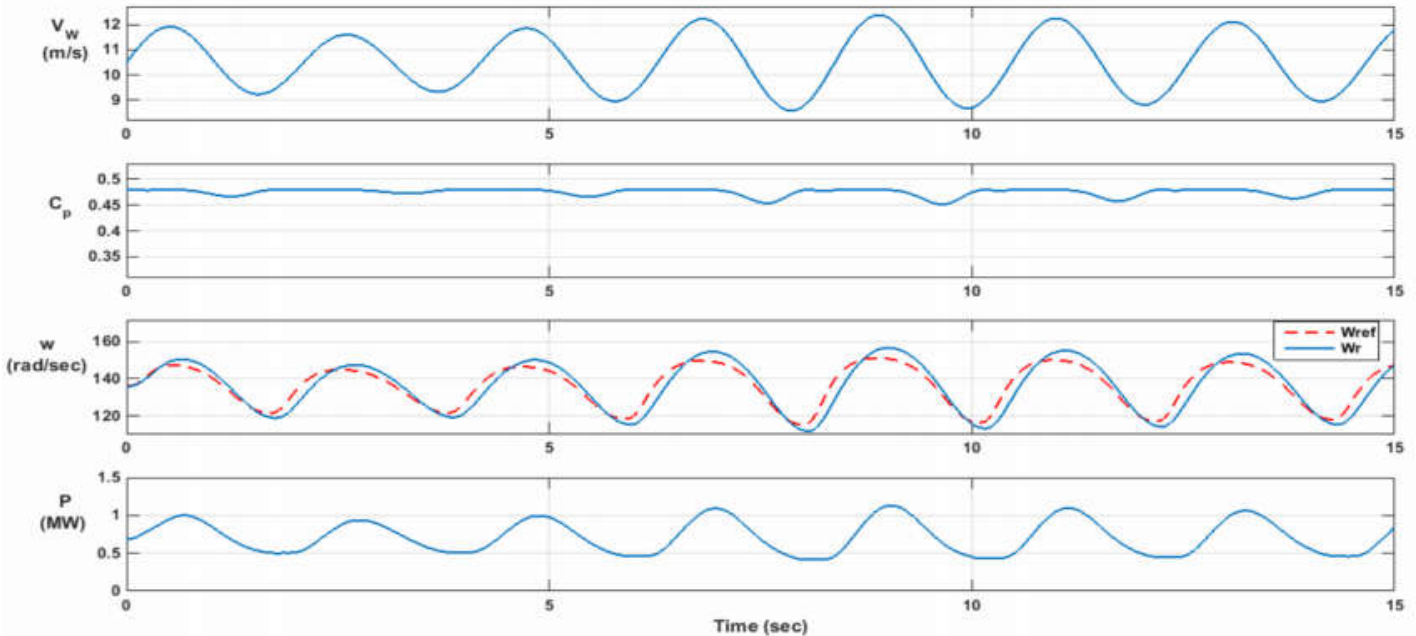

Fig. 12: $V_{w}, C_{p}, W$ and $P$ plots in the case of the wind velocity has sinusoidal changes.
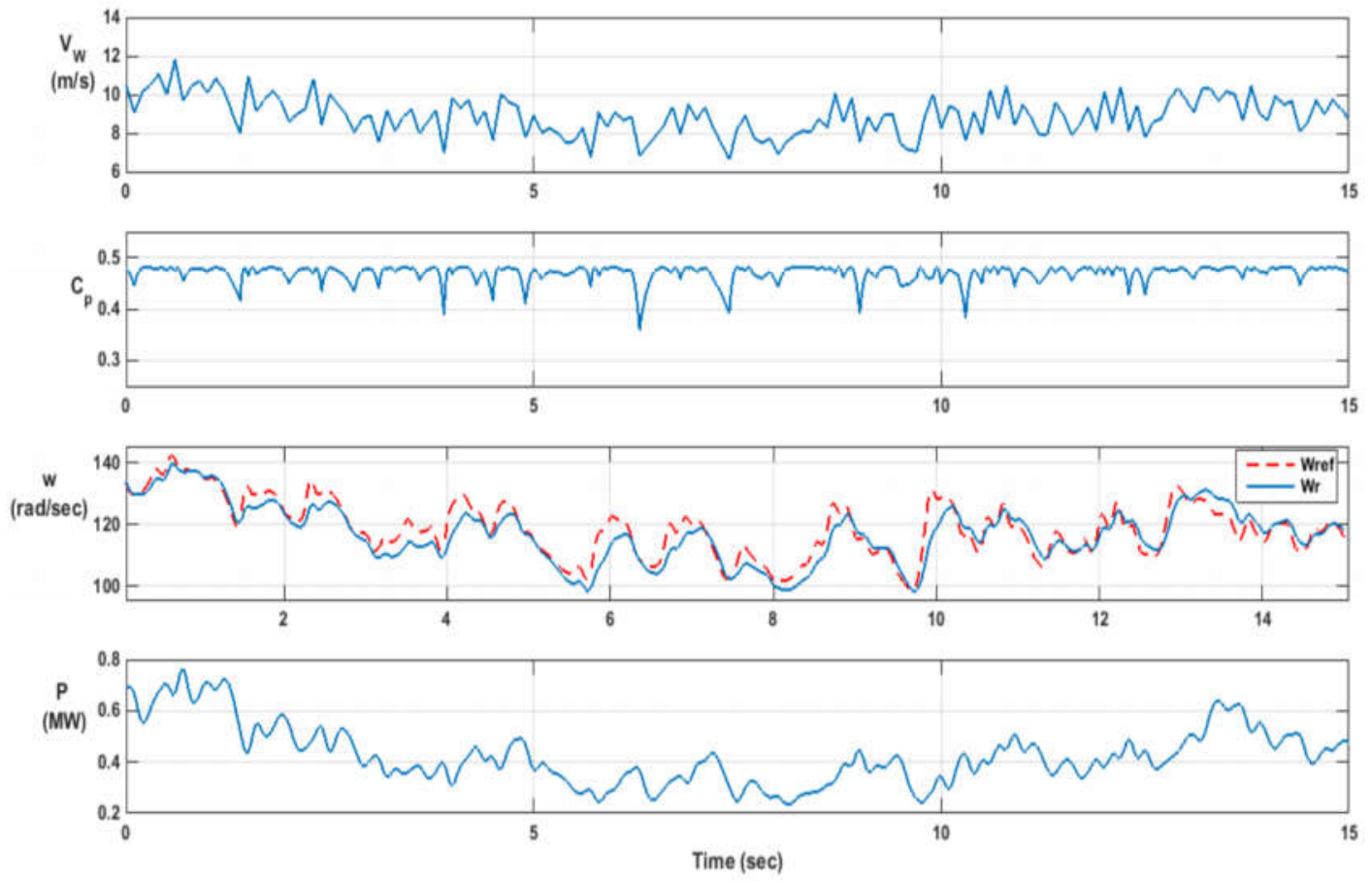

Fig. 13: $V_{w}, C_{p}, W$ and $P$ plots in the case that the noisy wind velocity applied

\section{Conclusions}

In this study, an ANN-based MPPT controller for a variable speed wind power generation with doubly fed induction generator has been proposed to protect the wind-turbine generation system from oscillation. Basically, the torque characteristics of the windturbine is determined by the conditions of the wind velocity, thereby the algorithms that change the maximum power point location needs to be developed in order to face the maximum-power- point-tracking (MPPT) control problem to get the optimal efficiency from wind energy at different operating conditions. The proposed method uses fully connected recurrent neural network (RNN) trained online using real-time recurrent learning (RTRL) algorithm, to generate control command to reach the optimum speed of the rotor side converter in order to enable the system to move toward the maximum power point. From the simulation results, the validity of the proposed MPPT controller has been verified. 

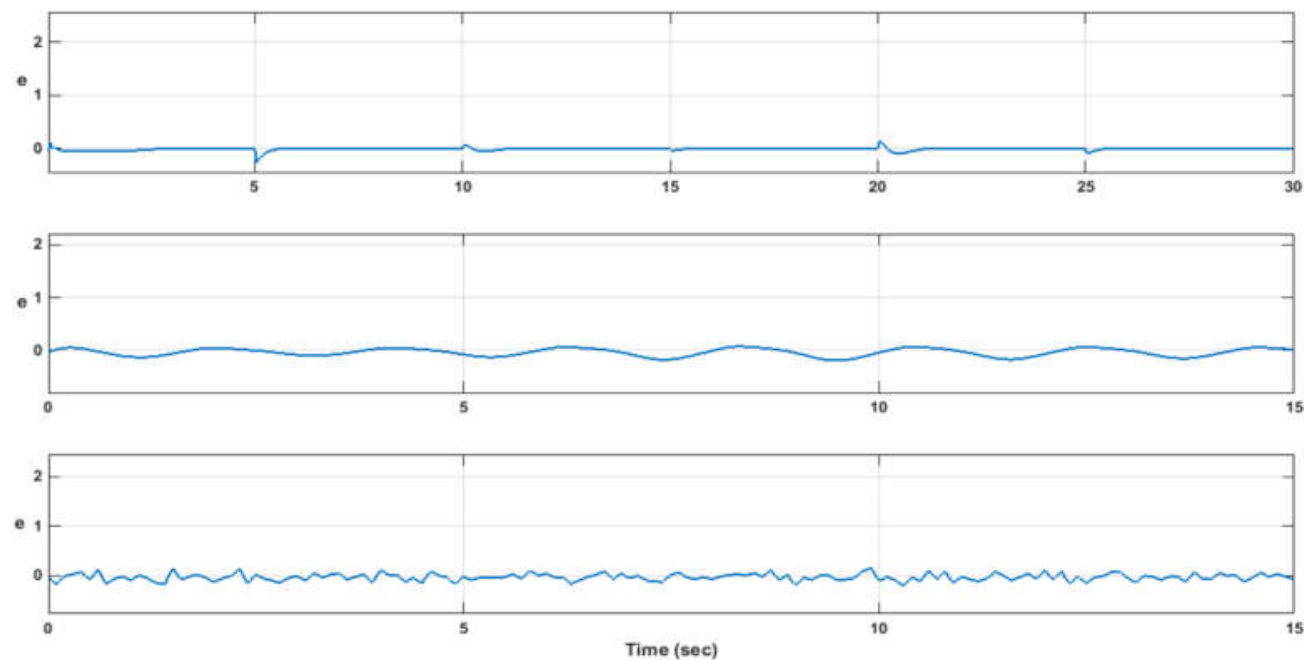

Fig. 14: Tracking error

\section{References}

[1] Tan K, Islam S. Optimum control strategies in energy conversion of PMSG wind turbine system without mechanical sensors. IEEE Trans Energy Convers, Vol 19, No. 2, pp. 392-399, 2004.

[2] Kolhe M, Joshi JC, Kothari DP. Performance analysis of a directly coupled photovoltaic water-pumping system. IEEE Trans Energy Convers, Vol.19, No.3, pp. 613-618, 2004.

[3] Andersen GK, Klumpner C, Kjaer SB, Blaabjerg F. A new green power inverter for fuel cells, IEEE 33rd annual power electron specialists conf, pp. 727-33, 2002.

[4] Mirecki A., Roboam X. and Richardeau F., Comparative study of maximum power strategy in wind turbines, IEEE Transactions on Energy Conversion, pp. 993-998, 2004.

[5] H. Li, K. L Shi, and P. G. McLaren, Neural network-based sensor-less maximum wind energy capture with compensated power coefficient, IEEE Trans. Industrial Applications, vol. 41, no. 6, pp. 1548-1156, 2005.

[6] Manganiello, P.; Ricco, M.; Petrone, G.; Monmasson, E.; Spagnuolo, G. Optimization of Perturbative PV MPPT Methods through Online System Identification, IEEE Trans. Ind. Electron., Vol. 61, 6812-6821, 2014.

[7] T. Shanthi, A.S. Vanmukhil, ANFIS controller based MPPT control of photovoltaic generation system, Research journal of applied sciences, Vol. 8, No. 7, pp. 375-382, 2013.

[8] Youngjong Ko, Holger Jedtberg, Giampaolo Buticchi, Marco Liserre, Topology and control strategy for accelerated lifetime test setup of DC-link capacitor of wind turbine converter, IEEE Applied Power Electronics Conference and Exposition (APEC) 2016, pp. 3629-3636, 2016.

[9] Y.-H. Liu, C.-L. Liu, J.-W. Huang, J.-H. Chen, Neural-network based maximum power point tracking methods for photovoltaic systems operating under fast changing environments, Solar Energy, Vol. 89, pp. 42-53, 2013.

[10] M.M. Atiqur Rahman, A.H.M.A. Rahim, An efficient wind speed sensor-less MPPT controller using adaptive neuro-fuzzy inference system, Advances in Electrical Engineering (ICAEE) 2015 International Conference, pp. 230-233, 2015.

[11] Soro S. Martin, Ahmed Chebak, Abderazak El Ouafi, Mustapha Mabrouki, An Efficient Fuzzy Logic Based MPPT Control Strategy for Multi-Source Hybrid Power System, 6th International Conference on Renewable and Sustainable Energy (IRSEC), pp. 1-8, 2018.

[12] F. Chekired, C. Larbes, D. Rekioua, F. Haddad, Implementation of a MPPT fuzzy controller for photovoltaic systems on FPGA circuit, Energy Procedia, vol. 6, pp. 541-549, 2011.

[13] R. Boukenoui, H. Salhi, R. Bradai, A. Mellit, A new intelligent MPPT method for stand-alone photovoltaic systems operating under fast transient variations of shading patterns, Solar Energy, vol. 124, pp. 124-142, 2015.

[14] R. Datta and V. T Tanganathan, A method of tracking the peak power points for a variable speed wind energy conversion system," IEEE 
Trans. Energy Conv., Vol 18, No.1, pp. 163168, 2003.

[15] Syafaruddin; Karatepe, E.; Hiyama, T. Artificial neural network-polar coordinated fuzzy controller based maximum power point tracking control under partially shaded conditions, IET Renewable Power Generation, pp. 239-253, 2009.

[16] Ahmed, J.; Salam, Z., An Improved Method to Predict the Position of Maximum Power Point during Partial Shading for PV Arrays, IEEE Trans. Ind. Inform., pp. 1378-1387, 2015.

[17] E. Koutroulis and K. Kalaitzakis, Design of a maximum power tracking system for windenergy-conversion applications, IEEE Trans. Ind . Electron., Vol. 53, No. 12, pp. 486-494, 2006.

[18] Rana Ahmed, A. Naaman, N. K. M'Sirdi, A. K. Abdelsalam, Y. G. Dessouky, Sensorless MPPT technique for PMSG micro wind turbines based on state-flow, International Conference on Renewable Energies for Developing Countries (REDEC), pp. 26-27, 2014.

[19] Siegfried Heier, Grid Integration of Wind Energy Conversion Systems, John Wiley \& Sons Ltd, 1998, ISBN 0-471-97143-X

[20] M. J. Z. Zadeh, S. H. Fathi, A new approach for photovoltaic arrays modeling and maximum power point estimation in real operating conditions, IEEE Transactions on Industrial Electronics, Vol. 64, pp. 9334-9343, 2017.

[21] Ansel Barchowsky, Jeffrey P. Parvin, Gregory F. Reed, Matthew J. Korytowski, and Brandon M. Grainger, Central and Distributed MPPT Systems under Varying Weather Conditions, Power and Energy Systems and Applications (PESA 2011), USA, November 2011

[22] R. Pena, J.C. Clare, G.M. Asher, Doubly fed induction generator using back-to-back PWM converters and its application to variable-speed wind-energy generation, IEEE Proc. Electr. Power Appl., Vol. 143, No. 3, 1996.

[23] Shuhui Li, Rajab Challoo, and Marty J. Nemmer, Comparative study of DFIG power control using stator-voltage and stator-flux oriented frames, IEEE power \& energy society general meeting, July 2009

[24] S. Haykin, Neural Networks and Learning Machines (3rd Edition), Prentice Hall, 2009.

[25] Mohsen Davoudi, Amin Kasiri far, Fully Connected Recurrent Neural Network MPPT Control Design For DFIG Wind Energy Conversion Systems, 2nd International
Conference on Knowledge based Engineering and Innovation (KBEI), 2015.

[26] M. Tsai, C. Tseng and Y. Hung, A novel MPPT control design for wind-turbine generation systems using neural network compensator, IECON 2012 - 38th Annual Conference on IEEE Industrial Electronics Society, Montreal, QC, pp. 3521-3526, 2012.

[27] S. Musunuri and H. L. Ginn, Comprehensive review of wind energy maximum power extraction algorithms, IEEE Power and Energy Society General Meeting, Detroit, MI, USA, pp. $1-8,2011$.

[28] J. S. Thongam, P. Bouchard, H. Ezzaidi and M. Ouhrouche, Artificial neural network-based maximum power point tracking control for variable speed wind energy conversion systems, IEEE Control Applications, (CCA) \& Intelligent Control, (ISIC), St. Petersburg, pp. 1667-1671, 2009.

[29] Chih-Hong Lin, Recurrent modified Elman neural network control of PM synchronous generator system using wind turbine emulator of PM synchronous servo motor drive, International Journal of Electrical Power \& Energy Systems Volume 52, Pages 143-160, 2013.

[30] Mohammad Rashed M. Altimania, MODELING OF DOUBLY-FED INDUCTION GENERATORS CONNECTED TO DISTRIBUTION SYSTEM BASED ON eMEGASim REAL-TIME DIGITAL SIMULATOR, University of Tennessee at Master of Engineering Thesis, Tennessee, May 2014.

[31] Daniil Naumetc, BUILDING THE ARTIFICIAL NEURAL NETWORK ENVIRONMENT-Artificial Neural Networks in plane control, Bachelor's thesis, Valkeakoski Automation Engineering, HAME University of Applied Science, Fall 2016.

[32] M.M. Atiqur Rahman, NEURAL NETWORK BASED MAXIMUM POWER POINT TRACKING AND CONTROL OF PMSG WIND SYSTEM, KING FAHD UNIVERSITY OF PETROLEUM \& MINERALS DHAHRAN, Electrical Engineering Department, September 2014. 